\title{
Loss of intraoperative neurological monitoring signals during flexed prone positioning on a hinged open frame during surgery for kyphoscoliosis correction: case report
}

\author{
Randall B. Graham, MD, ${ }^{1}$ Mathew Cotton, MS, ${ }^{2}$ Antoun Koht, MD, ${ }^{3}$ and Tyler R. Koski, MD ${ }^{4}$ \\ ${ }^{1}$ Methodist Brain and Spine Institute, Dallas, Texas; and 'Division of Intraoperative Neurophysiologic Monitoring, Department of \\ Neurology, ${ }^{3}$ Department of Anesthesiology, and ${ }^{4}$ Department of Neurological Surgery, Northwestern University Feinberg School \\ of Medicine, Chicago, Illinois
}

\begin{abstract}
Various complications of prone positioning in spine surgery have been described in the literature. Patients in the prone position for extended periods are subject to neurological deficits and/or loss of intraoperative signals due to compression neuropathies, but positioning-related spinal deficits are rare in the thoracolumbar deformity population. The authors present a case of severe kyphoscoliotic deformity with critical thoracolumbar stenosis in which, during the use of a hinged open frame in the prone position, complete loss of intraoperative neural monitoring signals occurred while the frame was flexed into kyphosis to facilitate exposure and instrumentation placement. When the frame was reset to a neutral position, evoked potentials returned to baseline and the operation proceeded without complications. This case represents, to the authors' knowledge, the first report of loss of evoked potentials due to an alteration of prone positioning on a hinged open frame. When positioning patients in such a manner, careful attention should be directed to intraoperative signals in patients with critical stenosis and kyphotic deformity.
\end{abstract}

https://thejns.org/doi/abs/10.3171/2018.1.SPINE17811

KEYWORDS intraoperative neurological monitoring; evoked potentials; adult spinal deformity; positioning; complications; surgical technique

$\mathrm{S}$ URGERY for adult spinal deformity (ASD) carries with it a risk of neurological complications. The rate of postoperative neurological deficits, particularly following operations involving three-column osteotomies, varies from $2.6 \%$ to $22.18 \% .^{3,12,13}$ Recent studies in large groups of patients have demonstrated the accuracy of intraoperative neural monitoring (IONM) in detecting intraoperative neurophysiological alterations and suggested its utility in preventing subsequent postoperative clinical neurological deficits. ${ }^{1,8,14,18,20,21}$ Multiple monitoring modalities, such as motor evoked potential (MEP), somatosensory evoked potential (SSEP), and both triggered and free-running electromyography (EMG) monitoring, are nearly universally used in surgery for ASD.

Prone positioning of patients for ASD surgery, although often overlooked, can be a significant source of complications. ${ }^{5,6,10,22}$ Most of the described prone positioning-related complications are related to myocutaneous and/or vascular compression over sustained periods during the intraoperative period (i.e., pressure sores, limb ischemia, etc.). ${ }^{6}$ Perhaps the most feared neurological positioningrelated complication is postoperative visual loss, which is thought to be related to posterior ischemic optic neuropathy. ${ }^{6,10}$ Other neurological complications are related to compression and/or traction of peripheral nerves, such as ulnar neuropathies and brachial plexus injuries.., 22

Spinal deficits due to prone positioning have been described extensively in patients with cervical and craniovertebral junction pathologies. ${ }^{2,15}$ Furthermore, IONM has been suggested as a modality for both surveillance and prevention of such injuries. ${ }^{2}$ To date, a spinal positioningrelated neurological complication with subsequent detection via IONM has never been described in the literature, particularly during manipulation of a specialized hinged open frame, which we use for many thoracolumbar spinal pathologies. This type of bedframe has a hinge near its midpoint that can be flexed into various degrees of kyphosis and lordosis, allowing the surgeon to adjust the patient's

ABBREVIATIONS ASD = adult spinal deformity; EMG = electromyography; IONM = intraoperative neural monitoring; MEP = motor evoked potential; SSEP = somatosensory evoked potential.

SUBMITTED July 22, 2017. ACCEPTED January 30, 2018.

INCLUDE WHEN CITING Published online June 22, 2018; DOI: 10.3171/2018.1.SPINE17811. 

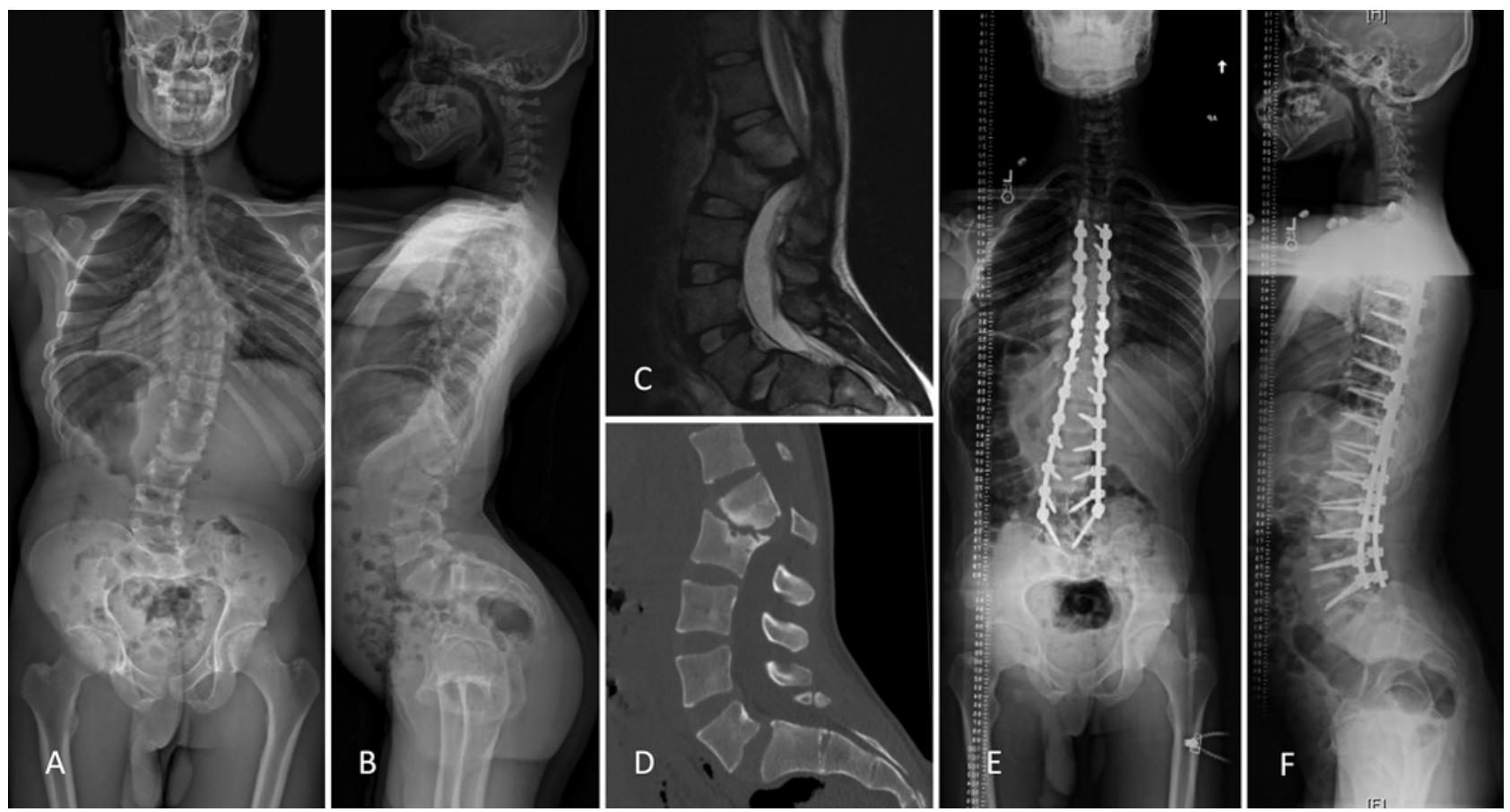

FIG. 1. Full-length standing anterior-posterior (A) and lateral (B) EOS radiographs depict a severe kyphoscoliotic deformity with lumbar and thoracic hyperlordosis. Sagittal T2-weighted MR image (C) and CT scan (D) demonstrate focal kyphosis at L1-2 with severe canal stenosis and dysplastic posterior elements. Postoperative anterior-posterior $(\mathbf{E})$ and lateral $(\mathbf{F})$ radiographs demonstrate interval correction of kyphoscoliosis following T4-L4 instrumented fusion and L1-2 transforaminal interbody fusion.

local and global alignment via changes to the frame's configuration. We typically position the patient onto the bed in a "neutral" position and then adjust the frame into kyphosis, which brings the patient's spine closer to the surface, thus facilitating exposure, instrumentation placement, osteotomies, and decompression. Then, prior to placing rods, the frame is adjusted back to neutral or into a lordotic configuration, which facilitates closure of the osteotomies and restoration of lordosis in certain patients. In this report, we present the unique case of a patient with significant thoracolumbar kyphoscoliosis who, at first, had recordable lower-extremity SSEPs and MEPs that were completely lost upon adjusting the bedframe into kyphosis.

\section{Case Report}

History and Examination

A 30-year-old otherwise healthy man presented to the neurosurgical clinic with about a 5-year history of progressive axial middle back pain. He then began to experience bilateral lower-extremity pain, numbness, and paresthesias in both feet, difficulty ambulating, and bilateral lower-extremity weakness. His medical and surgical histories were otherwise unremarkable and he was never diagnosed with scoliosis or any other spinal condition during childhood or adolescence.

Physical examination revealed normal muscle tone and strength in his bilateral upper and lower extremities. He was also found to have normal deep tendon reflexes and no long tract signs but an impaired tandem gait. Examination of his standing posture revealed significant clinical deformity with a palpable and visible kyphosis at the thoracolumbar junction and profound hyperlordosis in both the lumbosacral and midthoracic areas.

\section{Radiological Evaluation}

Standing anterior-posterior and lateral full-body threedimensional EOS scans (EOS Imaging) revealed an atypical thoracolumbar kyphoscoliosis with both lumbar and thoracic hyperlordosis (Fig. 1A and B). MRI of the lumbar spine demonstrated subluxation at L1-2 with critical canal stenosis due to ventral compression (Fig. 1C). CT scanning also revealed dysplasia of the posterior elements at the kyphotic level (Fig. 1D).

\section{Operation and Operative Course}

After induction of general anesthesia, SSEP and MEP monitoring was set up according to a standard protocol used in our institution. SSEP signals were monitored via stimulation of the median and posterior tibial nerves and recording from cortical and subcortical Erb's points. MEPs were obtained via transcranial motor stimulation and recording from the upper and lower extremities. The patient was positioned prone on a specialized hinged open frame table (ProAxis Spinal Surgery Table; Mizuho OSI) in its neutral configuration (Fig. 2B). Of note, prepositioning SSEP and MEP testing was not done, as it was the surgeon's judgment to obtain baseline signals in the prone position as soon as the patient was placed in the prone position. Prone baseline SSEP and MEP tracings were obtained, and the table was then adjusted into a kyphotic configuration to assist with exposure and instrumenta- 

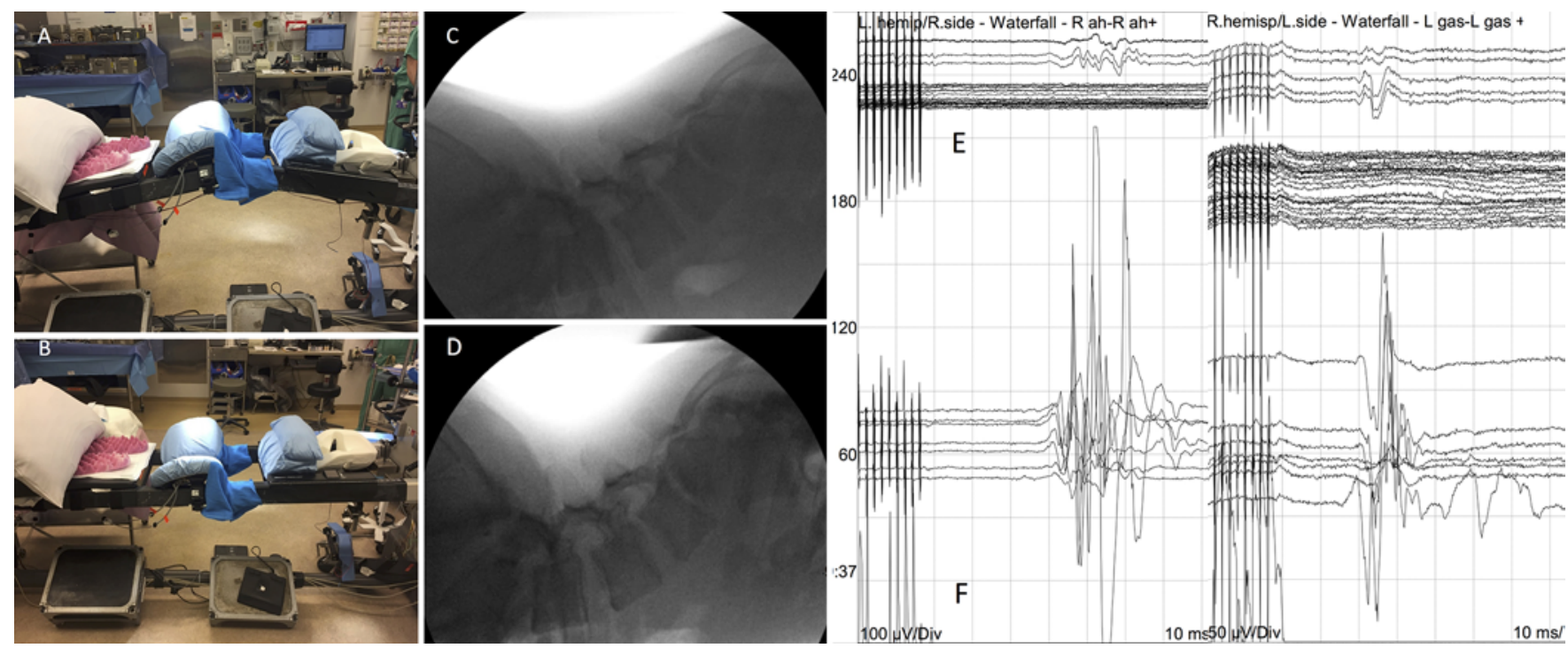

FIG. 2. The ProAxis hinged open spinal surgery frame in kyphotic (A) and neutral (B) configurations. Postpositioning lateral fluoroscopic images of the patient's lumbar spine with the frame in kyphotic (C) and neutral (D) positions. In kyphosis, there was nearcomplete loss of MEPs $(\mathbf{E})$. When the patient was returned to a neutral position, there was return of MEPs (F). Figure is available in color online only.

tion of the lower lumbar segments (Fig. 2A). Fluoroscopic images were obtained to make sure the table adjustment would not worsen the patient's subluxation (Fig. 2C). Shortly after the table and patient were adjusted into kyphosis, the bilateral lower-extremity MEP and SSEP signals dropped out entirely (Fig. 2E). Immediate attention was directed to the patient's blood pressure, which was appropriate on both cuff and intraarterial readings. The table was thus adjusted back into a neutral position, and after a few minutes, the patient's bilateral lower-extremity signals returned to their postpositioning baseline (Fig. $2 \mathrm{~F})$. There was additionally some discussion between the surgical and anesthesia teams regarding the potential effects of inhaled anesthetic agents on the patient's signals, but, given the rapid return of MEPs upon restoring the patient's positioning to neutral and the fact that only lowerextremity MEPs were affected (as opposed to a global loss of signals), it was decided that the etiology of signal loss was not pharmacological.

It was then decided to proceed with the procedure. The patient underwent a T4-L4 instrumented posterior spinal fusion, with a wide laminectomy across the thoracolumbar junction to remove the dysplastic posterior elements across those segments. Due to his critical stenosis and initial issues with positioning, it was decided to perform this decompression prior to instrumentation. The patient was noted intraoperatively to have severe stenosis across this kyphotic area, and, in fact, upon completion of the dorsal decompression, the patient's intraoperative signals were noted to immediately improve compared to baseline. The ProAxis frame was then flexed back into the kyphotic position to facilitate the placement of instrumentation across the hyperlordotic lower segments. Repeated SSEP and MEP signals following adjustment back into the kyphotic configuration did not result in any further signs of neurological deterioration as occurred during positioning.
Freehand placement of pedicle screw instrumentation was then carried out from T4 to L4, and complete bilateral L1-2 facetectomies with ligamentous release (i.e., posterior column osteotomies) were performed. Despite this, the kyphotic and subluxated L1-2 segment was still quite stiff. Thus, both exiting nerve roots were identified, and a bilateral transforaminal approach to the anterior disc space was performed with an aggressive anterior discectomy and arthrodesis. After this maneuver, the kyphotic subluxation became more mobile.

Two 6-0 cobalt chromium rods were then cut and bent into lordosis at the distal end and left mostly neutral at the proximal end with the goal of correcting the focal kyphotic deformity while also eliminating the hyperlordosis in the thoracic spine. The rods were therefore fixed from L4 up to L2 with screw caps and then, using bilateral simultaneous cantilever movements, the now-released L1 segment was pushed anteriorly, correcting the focal deformity. Using sequential reduction towers bilaterally, the rodding proceeded in a simultaneous fashion up to T4, reducing the initial thoracic hyperlordosis to a slightly kyphotic configuration.

\section{Postoperative Course}

After completion of the operation, the patient awoke from general anesthesia with full strength in his bilateral lower extremities. He had an overall uneventful postoperative course and was discharged to his home on postoperative day 5 . Note his postoperative long-cassette anteriorposterior and lateral standing "scoliosis" radiographs (Fig. $1 \mathrm{E}$ and $\mathrm{F})$.

\section{Discussion}

Surgery for ASD can be fraught with complications, the most feared of which is permanent neurological injury. As 
discussed previously, neurophysiological monitoring has become an effective method for intraoperative neurological surveillance and prevention of potential postoperative deficits. Although various complications from prone positioning in complex spinal surgery have been well documented, thoracolumbar spine-related neurological deficits from positioning have not been described. Additionally, to the best of our knowledge, there have been no reports of intraoperative signal alterations due to adjustments of hinged open spinal surgery frames.

It is important to reiterate the role of intraoperative neurophysiological monitoring in the care of this particular patient. If preadjustment prone baseline signals in the neutral configuration had not been obtained and the drop in those signals during kyphotic adjustment had not been noticed and addressed, the patient could have suffered irreversible neurological damage. Although false-negative results with IONM have been reported to occur in surgery for spinal deformity, they are exceedingly rare, and IONM remains highly sensitive and specific in the detection of impending intraoperative neurological insults. ${ }^{17,21}$ In cases of severe spinal deformities or patients with multiple previous spinal operations, loss of signals is potentially the result of vascular stretching and/or kinking that occurs along with significant intraoperative alterations to spinal alignment. This may affect blood flow at different points of the spinal canal and branches of spinal arteries and their perforators, resulting in focal ischemia. This is likely especially true in patients with severe longstanding kyphosis, in which the anterior spinal artery can be stretched chronically over the kyphotic segments. Adding kyphosis via flexion of the hinged frame can therefore stretch the artery even more, resulting in interruption of blood flow and ischemia. Conversely, surgeons should be aware that aggressive correction of such a focal kyphosis can kink the already stretched vessel, also resulting in ischemia and loss of MEP signals.

As we mentioned, prepositioning SSEP and MEP signal testing in the supine position was not performed in this particular case. The surgeon's primary concern at the start of this procedure was the effect of kyphosis adjustment using the hinged frame on the patient's highly stenotic subluxated segments, so meticulous attention was paid to the prone neutral versus prone kyphotic signals and fluoroscopic appearance. In retrospect, however, obtaining baseline signals in the supine position could have potentially added useful information. For example, if there was even a subtle decrease in signals between supine and prone neutral positioning, this could have served as a warning to avoid flexing the bed into further kyphosis.

When performing such complex spinal operations, it is imperative to have seamless communication among the surgical, anesthetic, and neural monitoring teams. It is also essential to have set protocols for dealing with intraoperative declines in IONM signals.? Technical, physiological, pharmacological, positional, and surgical factors can all cause changes in evoked potentials, and when such a change occurs, it is imperative to first make sure it is reproducible by excluding technical causes, such as issues with impedance or disconnections of leads or wires. The monitoring team should then distinguish between global and regional changes. A global change is more likely due to a pharmacological or physiological change (such as alterations in blood pressure or anesthetic agents). In contrast, a sudden focal change in evoked potentials (especially during a specific surgical or, in this case, positional maneuver) should raise attention to a positional or surgical cause. In such instances, the surgical team should identify and reverse the offending maneuver as quickly as possible, while the anesthetic team provides the physiological support necessary to boost blood circulation to the affected area and prevent longstanding ischemia and resultant neurological deficit.

It is also important to highlight the use of the hinged open spinal surgery bedframe. Such flexibility with positioning can facilitate exposure, instrumentation, and manipulation at deep lordotic segments. Furthermore, hinged bedframes are also useful in providing distraction of spinal segments when in the kyphotic position and compression when in the lordotic position. This is a powerful tool that can facilitate posterior access to the disc space and aid in opening and closing spinal osteotomies. Our case illustrates, however, that such versatility can bring about significant neurological risk in patients with severe stenosis due to kyphotic deformity. We therefore recommend exercising caution when using adjustable bedframes in the treatment of patients with such pathologies and to strongly consider obtaining preadjustment SSEPs and MEPs in the setting of severe kyphoscoliosis with stenosis.

Although, to our knowledge, this is the first report of complete MEP loss upon alteration of positioning using such a flexed open frame, there have been many reports of positioning-related changes in evoked potentials. Appel et al. studied 380 patients undergoing cervical spinal operations and found that head position can have a dramatic impact on intraoperative signals. ${ }^{2}$ Similarly, Schwartz et al. illustrated the use of SSEP and MEP monitoring to identify positioning-related neurological injuries during anterior cervical surgery, although these injuries were mostly related to brachial plexus stretching or ulnar nerve compression. ${ }^{19}$ As mentioned earlier, there have been many reports of compression-related myocutaneous and peripheral nerve complications, including compartment syndrome and ischemic optic neuropathy related to positioning for spinal operations. . $^{4-10,22}$ ASD surgery presents a unique set of risks with regard to intraoperative neurological injury, particularly in patients with preexisting deficits such as the one described in this case report. ${ }^{13}$ Risk factors for neurological injuries in spinal deformity surgery include magnitude of curvature correction, use of three-column osteotomies, revision surgery, and hyperkyphosis, par-

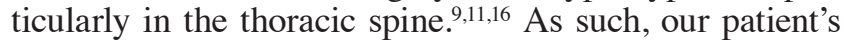
focal kyphotic deformity with severe stenosis likely increased his risk of a neurological complication. Given the patient's more recent bilateral lower-extremity symptoms, one could argue that the compression from his kyphosis was beginning to reach a critical point and even the slight exacerbation from flexion of the hinged bedframe was enough to cause further deficit.

\section{Conclusions}

To our knowledge, this is the first reported case of loss 
of intraoperative neurophysiological monitoring signals due to prone position manipulation using a hinged spinal surgery open frame for correction of ASD. Although positioning-related complications are not well described in thoracolumbar deformity surgery, it should be suspected if an unexplained alteration in signals occurs prior to making incision. If use of a hinged open spinal frame is planned, careful attention to evoked potentials is absolutely required and obtaining preadjustment baseline signals is strongly recommended, particularly in the setting of stenosis across a focal kyphotic deformity.

\section{References}

1. Agarwal N, Hamilton DK, Ozpinar A, Choi P, Hart R, Yaylali I: Intraoperative neurophysiologic monitoring for adult patients undergoing posterior spinal fusion. World Neurosurg 99:267-274, 2017

2. Appel S, Korn A, Biron T, Goldstein K, Rand N, Millgram $\mathrm{M}$, et al: Efficacy of head repositioning in restoration of electrophysiological signals during cervical spine procedures. J Clin Neurophysiol 34:174-178, 2017

3. Bianco K, Norton R, Schwab F, Smith JS, Klineberg E, Obeid I, et al: Complications and intercenter variability of three-column osteotomies for spinal deformity surgery: a retrospective review of 423 patients. Neurosurg Focus 36(5):E18, 2014

4. Bronson WH, Forsh D, Qureshi SA, Deiner SG, Weisz DJ, Hecht AC: Evolving compartment syndrome detected by loss of somatosensory- and motor-evoked potential signals during cervical spine surgery. Orthopedics 35:e1453-e1456, 2012

5. Chung I, Glow JA, Dimopoulos V, Walid MS, Smisson HF, Johnston KW, et al: Upper-limb somatosensory evoked potential monitoring in lumbosacral spine surgery: a prognostic marker for position-related ulnar nerve injury. Spine J 9:287-295, 2009

6. DePasse JM, Palumbo MA, Haque M, Eberson CP, Daniels $\mathrm{AH}$ : Complications associated with prone positioning in elective spinal surgery. World J Orthop 6:351-359, 2015

7. Gonzalez AA, Jeyanandarajan D, Hansen C, Zada G, Hsieh PC: Intraoperative neurophysiological monitoring during spine surgery: a review. Neurosurg Focus 27(4):E6, 2009

8. Gunnarsson T, Krassioukov AV, Sarjeant R, Fehlings MG: Real-time continuous intraoperative electromyographic and somatosensory evoked potential recordings in spinal surgery: correlation of clinical and electrophysiologic findings in a prospective, consecutive series of 213 cases. Spine (Phila Pa 1976) 29:677-684, 2004

9. Iorio JA, Reid P, Kim HJ: Neurological complications in adult spinal deformity surgery. Curr Rev Musculoskelet Med 9:290-298, 2016

10. Kamel I, Barnette R: Positioning patients for spine surgery: Avoiding uncommon position-related complications. World J Orthop 5:425-443, 2014

11. Kamerlink JR, Errico T, Xavier S, Patel A, Patel A, Cohen A, et al: Major intraoperative neurologic monitoring deficits in consecutive pediatric and adult spinal deformity patients at one institution. Spine (Phila Pa 1976) 35:240-245, 2010

12. Kelly MP, Lenke LG, Shaffrey CI, Ames CP, Carreon LY, Lafage V, et al: Evaluation of complications and neurological deficits with three-column spine reconstructions for complex spinal deformity: a retrospective Scoli-RISK-1 study. Neurosurg Focus 36(5):E17, 2014
13. Lenke LG, Fehlings MG, Shaffrey CI, Cheung KM, Carreon L, Dekutoski MB, et al: Neurologic outcomes of complex adult spinal deformity surgery: results of the prospective, multicenter Scoli-RISK-1 study. Spine (Phila Pa 1976) 41:204-212, 2016

14. Nuwer MR, Emerson RG, Galloway G, Legatt AD, Lopez J, Minahan R, et al: Evidence-based guideline update: intraoperative spinal monitoring with somatosensory and transcranial electrical motor evoked potentials. J Clin Neurophysiol 29:101-108, 2012

15. Ofiram E, Lonstein JE, Skinner S, Perra JH: "The disappearing evoked potentials": a special problem of positioning patients with skeletal dysplasia: case report. Spine (Phila Pa 1976) 31:E464-E470, 2006

16. Qiu Y, Wang S, Wang B, Yu Y, Zhu F, Zhu Z: Incidence and risk factors of neurological deficits of surgical correction for scoliosis: analysis of 1373 cases at one Chinese institution. Spine (Phila Pa 1976) 33:519-526, 2008

17. Raynor BL, Padberg AM, Lenke LG, Bridwell KH, Riew KD, Buchowski JM, et al: Failure of intraoperative monitoring to detect postoperative neurologic deficits: a 25-year experience in 12,375 spinal surgeries. Spine (Phila Pa 1976) 41:1387-1393, 2016

18. Schwartz DM, Auerbach JD, Dormans JP, Flynn J, Drummond DS, Bowe JA, et al: Neurophysiological detection of impending spinal cord injury during scoliosis surgery. $\mathbf{J}$ Bone Joint Surg Am 89:2440-2449, 2007

19. Schwartz DM, Sestokas AK, Hilibrand AS, Vaccaro AR, Bose B, Li M, et al: Neurophysiological identification of position-induced neurologic injury during anterior cervical spine surgery. J Clin Monit Comput 20:437-444, 2006

20. Sutter M, Eggspuehler A, Grob D, Jeszenszky D, Benini A, Porchet F, et al: The diagnostic value of multimodal intraoperative monitoring (MIOM) during spine surgery: a prospective study of 1,017 patients. Eur Spine J 16 (Suppl 2):S162S170, 2007

21. Thirumala PD, Crammond DJ, Loke YK, Cheng HL, Huang J, Balzer JR: Diagnostic accuracy of motor evoked potentials to detect neurological deficit during idiopathic scoliosis correction: a systematic review. J Neurosurg Spine 26:374383, 2017

22. Uribe JS, Kolla J, Omar H, Dakwar E, Abel N, Mangar D, et al: Brachial plexus injury following spinal surgery. J Neurosurg Spine 13:552-558, 2010

\section{Disclosures}

The authors report no conflict of interest concerning the materials or methods used in this study or the findings specified in this paper.

\section{Author Contributions}

Conception and design: all authors. Acquisition of data: Graham. Analysis and interpretation of data: Graham. Drafting the article: Graham. Critically revising the article: all authors. Reviewed submitted version of manuscript: all authors. Approved the final version of the manuscript on behalf of all authors: Graham.

\section{Correspondence}

Randall B. Graham: Methodist Brain and Spine Institute, Dallas, TX.randall.b.graham@gmail.com. 